\section{(1) \\ CrossMark}

\title{
Low-dose corticosteroid use and mortality in severe community-acquired pneumonia patients
}

\author{
Takashi Tagami ${ }^{1,2}$, Hiroki Matsui ${ }^{1}$, Hiromasa Horiguchi ${ }^{3}$, Kiyohide Fushimi ${ }^{4}$ and \\ Hideo Yasunaga ${ }^{1}$
}

Affiliations: ${ }^{1}$ Dept of Clinical Epidemiology and Health Economics, School of Public Health, Graduate School of Medicine, The University of Tokyo, Tokyo, Japan. ${ }^{2}$ Dept of Emergency and Critical Care Medicine, Nippon Medical School, Tokyo, Japan. ${ }^{3}$ Dept of Clinical Data Management and Research, Clinical Research Center, National Hospital Organization Headquarters, Tokyo, Japan. ${ }^{4}$ Dept of Health Informatics and Policy, Tokyo Medical and Dental University, Graduate School of Medicine, Tokyo, Japan.

Correspondence: Takashi Tagami, Dept of Clinical Epidemiology and Health Economics, School of Public Health, Graduate School of Medicine, The University of Tokyo, 7-3-1 Hongo, Bunkyo-ku, Tokyo 1138555, Japan. E-mail: t-tagamianms.ac.jp

ABSTRACT The relationship between low-dose corticosteroid use and mortality in patients with severe community-acquired pneumonia (CAP) remains unclear.

6925 patients with severe CAP who received mechanical ventilation with or without shock (defined as use of catecholamines) at 983 hospitals were identified using a Japanese nationwide administrative database. The main outcome measure was 28-day mortality.

2524 patients with severe CAP who received catecholamines were divided into corticosteroid ( $\mathrm{n}=631$ ) and control $(\mathrm{n}=1893)$ groups. The 28 -day mortality was significantly different between corticosteroid and control groups (unmatched: $24.6 \%$ versus $36.3 \%$, $\mathrm{p}<0.001$; propensity score-matched: $25.3 \%$ versus $32.6 \%$, $\mathrm{p}=0.01$; inverse probability-weighted: $27.5 \%$ versus $34.2 \%, \mathrm{p}<0.001) .4401$ patients with severe CAP who did not receive catecholamines were also divided into corticosteroid $(n=1112)$ and control $(n=3289)$ groups. The 28-day mortality was not significantly different between corticosteroid and control groups in propensity score-matched analyses (unmatched: $16.0 \%$ versus $19.4 \%, \mathrm{p}=0.01$; propensity score-matched: $17.7 \%$ versus $15.6 \%, \mathrm{p}=0.22$; inverse probability-weighted: $18.8 \%$ versus $18.2 \%, \mathrm{p}=0.44$ ).

Low-dose corticosteroid use may be associated with reduced 28-day mortality in patients with septic shock complicating CAP.

@ERSpublications

Low-dose corticosteroids in severe CAP patients was associated with better prognosis only in those with septic shock http://ow.ly/AWqZW

For editorial comments see Eur Respir J 2015; 45: 305-307 [DOI: 10.1183/09031936.00225414].

Received: May 012014 | Accepted after revision: Aug 152014 | First published online: Oct 162014

Support statement: H. Horiguchi, K. Fushimi and H. Yasunaga received grant support from the Ministry of Health, Labour and Welfare of Japan (Research on Policy Planning and Evaluation, grant no. H25-Policy-010). The funders had no role in the execution of this study or the interpretation of the results.

Conflict of interest: Disclosures can be found alongside the online version of this article at erj.ersjournals.com 


\section{Introduction}

Community-acquired pneumonia (CAP) is a common and serious infectious disease, and is one of the leading causes of death worldwide $[1,2]$. The mortality rate is low in most ambulatory patients with CAP, but is $\sim 40 \%$ in patients with severe CAP who require intensive care, despite use of effective antibiotic therapy $[1,3]$. Effective additional therapy to reduce this high mortality rate has been sought for several decades [4].

Several previous reports suggested that excessive systemic inflammatory responses and/or relative adrenal insufficiency may lead to poor clinical outcomes in patients with severe CAP and sepsis [5-11]. Corticosteroids are potent inhibitors of inflammation, and are used to treat adrenal insufficiency [10]. However, a previous study found that short-term high-dose corticosteroid use had negative effects in patients with respiratory failure, including those with CAP [12]. There has been longstanding debate regarding the benefit of low-dose corticosteroid use in patients with CAP, but no consensus has been reached [4, 13-20]. Recent meta-analyses of randomised controlled trials found that even though low-dose corticosteroid use may not be beneficial to the overall population of patients with CAP, it may have a beneficial effect on mortality in patients with severe CAP and/or acute respiratory distress [13, 21-23]. However, recent small observational studies that focused on patients with severe CAP reported conflicting results [24-26]. Therefore, the effect of low-dose corticosteroid use on mortality in critically ill patients with severe CAP remains unclear.

We hypothesised that low-dose corticosteroid use may reduce mortality in patients with severe CAP. The purpose of the current study was to evaluate this hypothesis using a large, nationwide dataset available through the Japanese Diagnosis Procedure Combination (DPC) database.

\section{Materials and methods}

This study was approved by the Institutional Review Board of the University of Tokyo Hospital (Tokyo, Japan). Requirement for informed patient consent was waived because of the anonymous nature of the data.

\section{Data source and patient selection}

The DPC database includes administrative claims and abstract discharge data for all inpatients discharged from more than 1000 participating hospitals in Japan [27]. The database includes the following information for each patient: age; sex; primary diagnosis; comorbidities at admission and post-admission complications coded with International Classification of Diseases, 10th revision codes and written in Japanese; medical procedures, including types of surgery, coded with original Japanese codes; daily records of drug administration and devices used; length of stay; and discharge status. The dates of hospital admission, surgery, bedside procedures, drugs administered and discharge were recorded using a uniform data submission format [27].

The present study used data collected from July 1, 2010 to March 31, 2013. Only patients with the primary diagnosis recorded as CAP were included for the current study. To rule out hospital-acquired and ventilator-associated pneumonia, patients with pneumonia recorded as a comorbidity at admission or as a post-admission complication were not included [28]. The inclusion criteria were: 1) age $\geqslant 18$ years; 2) confirmed diagnosis of CAP (antibiotic therapy initiated on day 0 or 1), not including cases of "suspected pneumonia"; and 3) evidence of severe pneumonia, requiring mechanical ventilation within 7 days of admission [29, 30]. The exclusion criteria were: 1) pregnancy; 2) trauma, haematological malignancy, solid tumour, obstetric complications, or vascular disorders recorded as comorbidities at admission; 3) major surgery (under general anaesthesia) within 3 days after admission; 4) viral pneumonia (including influenza), aspiration pneumonia, HIV-related Pneumocystis jiroveci pneumonia, or tuberculous pneumonia; 5) hospitalisation in the same hospital within the preceding 90 days (to rule out healthcare-associated pneumonia); and 6) discharged within 2 days of admission [1, 3, 28, 31, 32]. Patients who received corticosteroids for $<3$ days, who received no corticosteroids in the early phase (within 7 days after admission), and who received high-dose corticosteroids were also excluded [12, 16, 21-23]. Low-dose corticosteroid use was defined as intravenous infusion of methylprednisolone $0.5-2.5 \mathrm{mg} \cdot \mathrm{kg}^{-1} \cdot \mathrm{day}^{-1}$ (or an equivalent dose of dexamethasone, hydrocortisone, prednisolone or betamethasone), and any higher dose was defined as high dose $[16,22]$.

\section{Variables and end-point}

In addition to the baseline characteristics at the time of admission, several other variables were evaluated in the current study. The Japan Coma Scale (JCS) [27] score at the time of admission was recorded for all patients. The JCS score correlates well with the Glasgow Coma Scale score, and a JCS score of 100 is equivalent to a Glasgow Coma Scale score of 6-9 [27]. The hospital type was categorised as academic or 
nonacademic. Hospital volume was defined as the mean annual number of corticosteroid prescriptions to CAP patients. Shock was defined as use of catecholamines (dopamine, dobutamine or noradrenaline) within 7 days of admission. The main endpoint was 28-day all-cause mortality.

\section{Statistical analysis \\ Propensity score analysis}

One-to-one matching was performed between patients in the corticosteroid and control groups using estimated propensity scores [32-34]. To estimate the propensity score, we fitted a logistic regression model for corticosteroid use as a function of patient and hospital factors including: age; sex; hospital type (academic or nonacademic) and hospital volume; JCS score; liver cirrhosis with a Child-Pugh score of 10-15 (Class C); coexisting lung disease (asthma or chronic obstructive pulmonary disease); evidence of pleural effusion with/without paracentesis or drainage; evidence of gastrointestinal ulcer bleeding; gastroscopy with/without a haemostatic procedure; intermittent haemodialysis and continuous renal replacement therapy after admission; type of catecholamine used (dopamine, dobutamine or noradrenaline); blood transfusion use; albumin use; antithrombin concentration use; nonsteroidal anti-inflammatory drug use; intravenous immunoglobulin use; sivelestat sodium use; initial use of two or more antibiotics; and each type of antibiotic used [1, 3, 27, 30, 35-39]. The C-statistic was used to evaluate the goodness of fit. One-to-one matched analysis using nearest-neighbour matching was performed based on the estimated propensity scores of the patients. A match occurred when a patient in the corticosteroid group had an estimated score within $0.25 \mathrm{SD}$ of a patient in the control group [27, 33]. Inverse probability-weighted estimators were used to examine the robustness of the results of the propensity matching analysis $[27,34]$.

\section{Instrumental variable analysis}

When hospitals have strongly consistent corticosteroid use patterns for severe pneumonia, it is assumed that decisions regarding corticosteroid use may be made independently of individual patient characteristics. In this situation, the hospital's corticosteroid use pattern may act as an instrumental variable, thereby setting the conditions for a "natural experiment" that allows an unbiased estimate of risk in patients with severe pneumonia $[27,34,40]$. A consistent result from the instrumental variable analysis may serve as useful confirmatory analysis for the propensity score analysis. Hospitals that used corticosteroids for $\geqslant 25.7 \%$ (mean value) of their pneumonia patients were classified as hospitals with a preference for corticosteroid use, and those that used corticosteroids for $<25.7 \%$ of their pneumonia patients were classified as hospitals without a preference for corticosteroid use. Estimated risk differences for 28-day mortality with $95 \%$ confidence intervals were calculated using the ivreg2 command in Stata/SE version 13.0 (StataCorp LP, College Station, TX, USA). The partial F-test was used to confirm that the hospital corticosteroid use pattern was not a weak instrument [40]. An F-statistic $>10$ suggests that the instrument is not weak [40].

Stratified analyses were performed for CAP patients with and without shock. Descriptive statistics are presented for all included patients (unmatched) and for propensity score-matched patients. Continuous variables were compared using the t-test, and categorical variables were compared using the Chi-squared test or Fisher's exact test. Kaplan-Meier plots with log-rank statistics were used to assess differences in survival between the propensity score-matched corticosteroid and control groups. Multivariable logistic regression analyses were performed for the unmatched, propensity score-matched and inverse probability-weighted groups to examine the associations between corticosteroid use and 28-day mortality. Values of $\mathrm{p}<0.05$ were considered statistically significant. All statistical analyses except the instrumental variable analysis were performed using IBM SPSS version 22 (IBM Corp., Armonk, NY, USA).

\section{Results}

During the 33-month study period, 33977 patients at 1163 hospitals were admitted with a primary diagnosis of pneumonia, including 14943 patients with CAP who required mechanical ventilation, of which 8018 were excluded from the current study (fig. 1). The remaining 6925 CAP patients who required mechanical ventilation at 983 hospitals were divided into CAP patients with shock $(n=2524)$ (table 1) and CAP patients without shock $(\mathrm{n}=4401)$ (table 2$)$. There was a significant difference in 28-day mortality between CAP patients with and without shock $(33.4 \%$ versus $18.5 \%, \mathrm{p}<0.001)$.

\section{Ventilated CAP patients with shock}

The 2524 CAP patients with shock were divided into corticosteroid $(n=631)$ and control $(n=1893)$ groups, from which 491 propensity score-matched pairs were generated. Calculation of the C-statistic showed that the goodness of fit was 0.75 in the propensity score-matched model. 
FIGURE 1 Patient selection. CAP: community-acquired pneumonia.

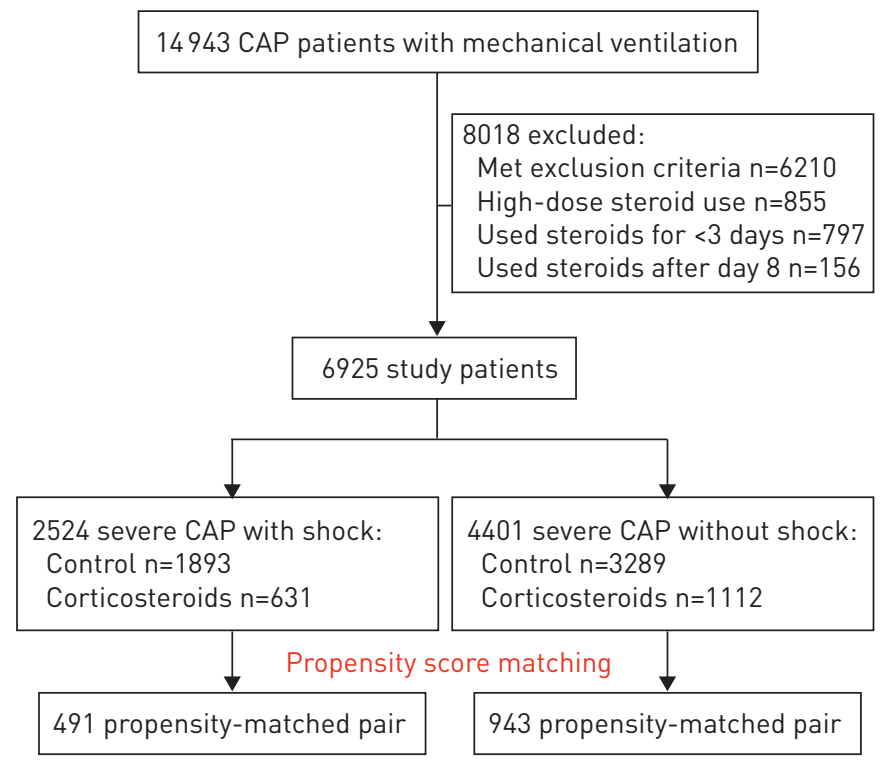

Table 1 shows the baseline characteristics of the unmatched corticosteroid and control groups $(n=2524)$ and the propensity score-matched groups $(n=982)$. In the unmatched groups, corticosteroid use was associated with admission to an academic hospital, and coexisting lung disease. The corticosteroid group received more noradrenaline, blood transfusions and blood products, and medications than the control group. The corticosteroid group received more fluoroquinolones, macrolides and anti-methicillin-resistant Staphylococcus aureus drugs, and were more likely to receive multiple concomitant antibiotics. The control group received more ampicillin/sulbactam. The baseline patient characteristics were similar between the propensity score-matched corticosteroid and control groups. The mean (quartile) duration of corticosteroid use was 8 (11) days.

The overall 28 -day mortality rate was $33.4 \%$ (843 out of 2524 ). The 28 -day mortality rate was significantly different between corticosteroid and control groups (unmatched: $24.6 \%$ versus $36.3 \%, \mathrm{p}<0.001$; propensity score-matched: $25.3 \%$ versus $32.6 \%, \mathrm{p}=0.01$; inverse probability-weighted: $27.5 \%$ versus $34.2 \%, \mathrm{p}<0.001$ ) (table 3). Kaplan-Meier survival curves for the propensity score-matched corticosteroid and control groups are shown in figure 2 (log-rank Chi-squared=6.99, $\mathrm{p}=0.008$ ). Multiple logistic regression analyses showed significant associations between corticosteroid use and lower 28-day mortality (table 4). In the instrumental variable model, the null hypothesis that there was no association between the pattern of hospital corticosteroid use and actual corticosteroid use was rejected $(\mathrm{p}<0.001, \mathrm{~F}$-statistic $=247.5)$. The estimated reduction in 28-day mortality associated with corticosteroid use was $27.4 \%$ (95\% CI $12.8-42.1$, $\mathrm{p}<0.001)$.

\section{Ventilated CAP patients without shock}

The 4401 CAP patients without shock were divided into corticosteroid $(n=1112)$ and control $(n=3289)$ groups, from which 943 propensity score-matched pairs were generated. Calculation of the C-statistic showed that the goodness of fit was 0.76 in the propensity score-matched model. Table 2 shows the baseline characteristics of the unmatched corticosteroid and control groups $(n=4401)$ and the propensity score-matched groups $(\mathrm{n}=1886)$. The mean (quartile) duration of corticosteroid use was 7 (7) days.

The overall 28 -day mortality rate was $18.5 \%$ (815 out of 4401 ). Although the 28 -day mortality was significantly different between the unmatched corticosteroid and control groups (16.0\% versus $19.4 \%$, $\mathrm{p}=0.01$ ), it was not significantly different between corticosteroid and control groups on propensity score-matched analysis $(17.7 \%$ versus $15.6 \%, \mathrm{p}=0.22)$ or inverse probability-weighted analysis $(18.8 \%$ versus $18.2 \%, \mathrm{p}=0.44$ ) (table 3 ). Multiple logistic regression analysis did not show a significant association between corticosteroid use and 28-day mortality (table 4). In the instrumental variable model, the null hypothesis that there was no association between the pattern of hospital corticosteroid use and actual corticosteroid use was rejected $(\mathrm{p}<0.001, \mathrm{~F}$-statistic $=306.8)$. Corticosteroid use was not associated with a significant estimated reduction in 28-day mortality (3.0\% reduction, 95\% CI -7.6-13.6, p=0.59). 
TABLE 1 Baseline characteristics in unmatched and propensity score-matched groups of patients with shock

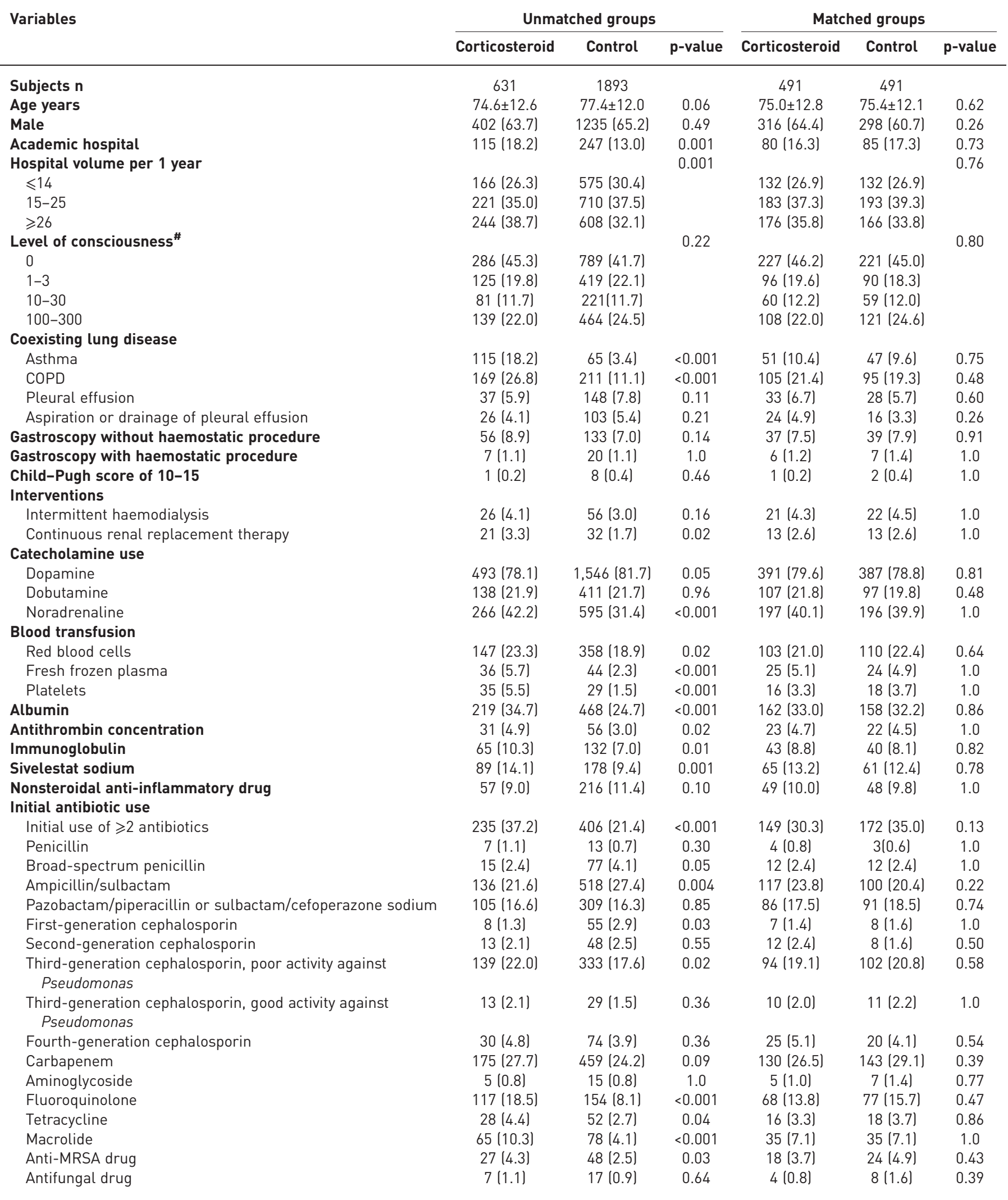

Data are presented as mean \pm SD or $\mathrm{n}(\%)$, unless otherwise stated. COPD: chronic obstructive pulmonary disease; MRSA: methicillin-resistant Staphylococcus aureus; \#: Japan Coma Scale score. 
TABLE 2 Baseline characteristics in unmatched and propensity score-matched groups of patients without shock

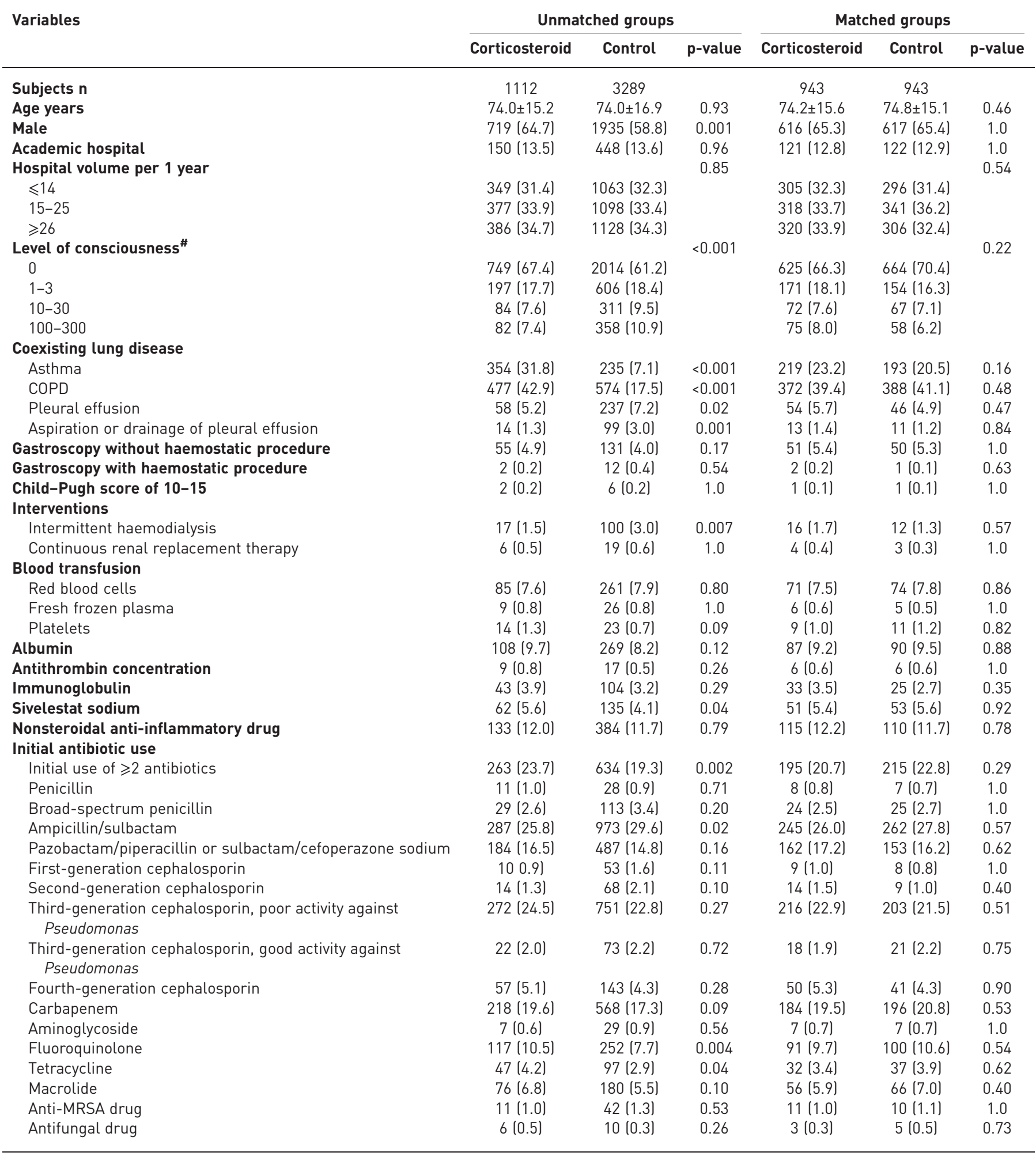

Data are presented as mean \pm SD or $n(\%)$, unless otherwise stated. COPD: chronic obstructive pulmonary disease. MRSA: methicillin-resistant Staphylococcus aureus. \#: Japan Coma Scale score. 


\section{Discussion}

Using a nationwide database, the results of this study suggest that there may be a significant association between low-dose corticosteroid use and 28-day mortality in patients with severe CAP and shock. However, this association was not found in patients with severe CAP without shock.

CAP is a heterogeneous infectious disease with various causes (bacterial, viral, fungal and parasitic), severities and immunological response patterns [1]. Several pro-inflammatory and anti-inflammatory cytokines are over synthesised and secreted into the systemic circulation during severe infections. The cytokine profiles of CAP patients vary according to the cause, severity and immunological response pattern of the disease 5 , and mortality is highest when both pro-inflammatory and anti-inflammatory cytokine levels are high [5]. Several clinical studies reported that the prognosis of CAP may differ according to the underlying condition of the patient $[1,3]$. Corticosteroid use was reported to be beneficial in HIV patients with $P$. jiroveci pneumonia 31, but not in patients with severe influenza A/H1N1 pneumonia [32]. Corticosteroid use may have a beneficial effect on mortality in patients with severe CAP and/or acute respiratory distress [13, 21, 22], but not in patients with mild CAP [21]. Therefore, we considered that evaluation of the efficacy of corticosteroid use for CAP should compare patient groups that are as homogeneous as possible. However, it is not easy to evaluate sufficiently large homogeneous populations of patients with CAP in prospective trials or single-centre observational studies. The current nationwide study analysed the clinical data of $>6900$ patients at 983 hospitals who were critically ill with CAP and received mechanical ventilation, with or without catecholamines. One of the strengths of the current study is the use of strict inclusion and exclusion criteria, enabling a large number of homogeneous patients with severe CAP to be reviewed. Additionally, we performed stratified analyses of patients with and without shock. We believe that this stratification is important when investigating patients with severe CAP who receive corticosteroids, because shock is an important contributing factor to 28-day mortality, and may respond to corticosteroid use $[10,23,26]$. The propensity score-matched approach is a powerful tool that attempts to construct a randomised experiment-like situation by comparing groups with similar observed characteristics, without specifying the relationships between confounders and outcomes. Although analysis of the baseline patient characteristics in the unmatched group showed more corticosteroid use in patients with more severe illness, one-to-one propensity score matching successfully balanced the characteristics between patient groups with and without corticosteroid use, including factors that have the potential to affect mortality or are known to affect mortality in patients with pneumonia, as described in the methods section [1, 3, 30, 35-39]. Our results suggest that patients with severe CAP and shock who received corticosteroids were less likely to die than similar patients who did not receive corticosteroids. This finding was robust with regard to the results obtained using probability-weighted, instrumental variable, and multiple logistic regression analyses. However, this relationship was not observed in patients with CAP without shock.

In patients with severe CAP, the relationship between corticosteroid use and mortality differed between patients with and without shock. This study clearly shows for the first time that benefits of low-dose corticosteroids in patients with severe CAP are related to the positive effects of these drugs on septic shock

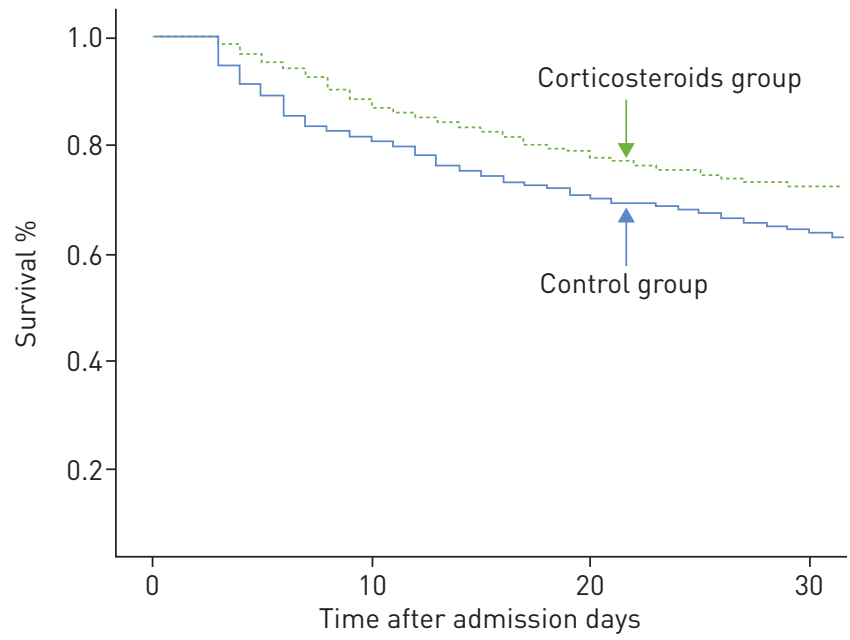

FIGURE 2 Kaplan-Meier survival curves for propensity score-matched patients with shock, treated with or without low-dose corticosteroids. In patients with shock who received catecholamines, there was a significant difference in survival between those who received corticosteroids and those who did not (log-rank Chi-squared=6.99, $\mathrm{p}=0.008$ ). 
TABLE 3 Comparisons of 28-day mortality rates between groups

Corticosteroid

Control

p-value

$\begin{array}{lc}\text { CAP patients with shock } & \\ \text { Unmatched groups } & 155 / 631(24.6) \\ \text { Propensity score-matched groups } & 124 / 491(25.3) \\ \text { Inverse probability-weighted groups } & 680 / 2477(27.5) \\ \text { CAP patients without shock } & \\ \text { Unmatched groups } & 178 / 1112(16.0) \\ \text { Propensity score-matched groups } & 167 / 943(17.7) \\ \text { Inverse probability-weighted groups } & 798 / 4242(18.8)\end{array}$

$688 / 1893$ (36.3)

$160 / 491(32.6)$

$872 / 2553(34.2)$

$637 / 3289(19.4)$

$147 / 943(15.6)$

$809 / 4456(18.2)$
$<0.001$

0.01

$<0.001$

0.01

0.24

0.44

Data are presented as $\mathrm{n} / \mathrm{N}(\%)$, unless otherwise stated. CAP: community-acquired pneumonia.

\begin{tabular}{lcc} 
TABLE 4 Logistic regression analyses for 28-day mortality for patients in corticosteroid groups \\
compared with control groups & p-value \\
\hline CAP patients with shock & Odds ratio (95\% Cl) & 0.005 \\
Unmatched groups & $0.72(0.57-0.91)$ & 0.01 \\
Propensity-matched groups & $0.68(0.50-0.92)$ & $<0.001$ \\
Inverse probability-weighted groups & $0.68(0.60-0.77)$ & 0.51 \\
CAP patients without shock & $1.1(0.87-1.3)$ & 0.18 \\
Unmatched groups & $1.2(0.92-1.5)$ & 0.58 \\
Propensity-matched groups & $1.0(0.92-1.2)$ &
\end{tabular}

Logistic regression analyses included the potential confounding variables listed in tables 1 and 2. CAP: community-acquired pneumonia.

rather than on CAP. Our results offer strong evidence to restrain from giving low-dose corticosteroids to patients with severe CAP when septic shock is not present. A prospective trial is required to confirm our results.

This study has some limitations. First, although the study used a nationwide database, it was retrospective and observational, without randomisation. Even though propensity score-matching was used to adjust for differences in baseline characteristics and disease severity, there may still be bias in the form of confounders that were not measured. Important examples of such confounders are the systemic and respiratory variables that represent the pathophysiology of severe pneumonia and are related to the prognosis, such as the Acute Physiology and Chronic Health Evaluation (APACHE) score, Sequential Organ Failure Assessment (SOFA) score, arterial oxygen tension/inspiratory oxygen fraction, ventilator settings, positive end-expiratory pressure level, and extravascular lung water. Unfortunately, these data were not available from the DPC database. Therefore, our results were additionally validated using instrumental variable analysis to compensate for these potential unmeasured confounders. Secondly, this study evaluated only early and prolonged low-dose corticosteroid use, because the results of previous studies suggested that short-duration, late-phase, and high-dose corticosteroid use are not beneficial in patients with severe CAP [12, 13, 18, 21]. Thirdly, the potential adverse effects of corticosteroid use, such as superinfection, hyperglycaemia and myopathy, could not be evaluated [18]. Fourthly, the DPC database does not include data regarding microbial aetiology. We tried to compensate for this limitation by evaluating all the antibiotics administered to patients. However, high proportions of patients received two or more antibiotics, carbapenem, piperacillin-tazobactam, anti-methicillin-resistant Staphylococcus aureus drugs, fluoroquinolone and fourth generation cephalosporins. Although these antibiotics are often used for the treatment of hospital-acquired pneumonia, only patients with the primary diagnosis of CAP at admission were included in the current study. We speculate that these antibiotics may have been used as empiric therapy in patients with severe CAP, as recommended by the current CAP treatment guidelines and sepsis guidelines [29, 30]. Finally, even though patients who had been hospitalised in the same hospital within the preceding 90 days were excluded, we cannot completely rule out the possibility that some patients with healthcare-associated pneumonia were included. 
In conclusion, the current study conducted propensity score and instrumental variable analyses using data from a large nationwide database, and found that low-dose corticosteroid use may result in improved 28-day prognosis in patients with severe CAP and shock, but not in patients with severe CAP without shock.

\section{References}

1 Wunderink RG, Waterer GW. Clinical practice. Community-acquired pneumonia. N Engl J Med 2014; 370: 543-551.

2 Angus DC, Linde-Zwirble WT, Lidicker J, et al. Epidemiology of severe sepsis in the United States: analysis of incidence, outcome, and associated costs of care. Crit Care Med 2001; 29: 1303-1310.

3 Fine MJ, Smith MA, Carson CA, et al. Prognosis and outcomes of patients with community-acquired pneumonia. A meta-analysis. JAMA 1996; 275: 134-141.

4 Marik P, Kraus P, Sribante J, et al. Hydrocortisone and tumor necrosis factor in severe community-acquired pneumonia. A randomized controlled study. Chest 1993; 104: 389-392.

5 Kellum JA, Kong L, Fink MP, et al. Understanding the inflammatory cytokine response in pneumonia and sepsis: results of the Genetic and Inflammatory Markers of Sepsis (GenIMS) Study. Arch Intern Med 2007; 167: $1655-1663$.

6 Headley AS, Tolley E, Meduri GU. Infections and the inflammatory response in acute respiratory distress syndrome. Chest 1997; 111: 1306-1321.

7 Puren AJ, Feldman C, Savage N, et al. Patterns of cytokine expression in community-acquired pneumonia. Chest 1995; 107: 1342-1349.

8 Salluh JI, Shinotsuka CR, Soares M, et al. Cortisol levels and adrenal response in severe community-acquired pneumonia: a systematic review of the literature. J Crit Care 2010; 25: 541.

9 Marik PE. Critical illness-related corticosteroid insufficiency. Chest 2009; 135: 181-193.

10 Marik PE, Pastores SM, Annane D, et al. Recommendations for the diagnosis and management of corticosteroid insufficiency in critically ill adult patients: consensus statements from an international task force by the American College of Critical Care Medicine. Crit Care Med 2008; 36: 1937-1949.

11 Salluh JI, Bozza FA, Soares M, et al. Adrenal response in severe community-acquired pneumonia: impact on outcomes and disease severity. Chest 2008; 134: 947-954.

12 Bernard GR, Luce JM, Sprung CL, et al. High-dose corticosteroids in patients with the adult respiratory distress syndrome. N Engl J Med 1987; 317: 1565-1570.

13 Cheng M, Pan ZY, Yang J, et al. Corticosteroid therapy for severe community-acquired pneumonia: a meta-analysis. Respir Care 2014; 59: 557-563.

14 Confalonieri M, Urbino R, Potena A, et al. Hydrocortisone infusion for severe community-acquired pneumonia: a preliminary randomized study. Am J Respir Crit Care Med 2005; 171: 242-248.

15 Garcia-Vidal C, Calbo E, Pascual V, et al. Effects of systemic steroids in patients with severe community-acquired pneumonia. Eur Respir J 2007; 30: 951-956.

16 Lamontagne F, Briel M, Guyatt GH, et al. Corticosteroid therapy for acute lung injury, acute respiratory distress syndrome, and severe pneumonia: a meta-analysis of randomized controlled trials. J Crit Care 2010; 25: 420-435.

17 Snijders D, Daniels JM, de Graaff CS, et al. Efficacy of corticosteroids in community-acquired pneumonia: a randomized double-blinded clinical trial. Am J Respir Crit Care Med 2010; 181: 975-982.

18 Meijvis SC, Hardeman H, Remmelts HH, et al. Dexamethasone and length of hospital stay in patients with community-acquired pneumonia: a randomised, double-blind, placebo-controlled trial. Lancet 2011; 377: 2023-2030.

19 Mikami K, Suzuki M, Kitagawa H, et al. Efficacy of corticosteroids in the treatment of community-acquired pneumonia requiring hospitalization. Lung 2007; 185: 249-255.

20 Fernandez-Serrano S, Dorca J, Garcia-Vidal C, et al. Effect of corticosteroids on the clinical course of community-acquired pneumonia: a randomized controlled trial. Crit Care 2011; 15: R96.

21 Nie W, Zhang Y, Cheng J, et al. Corticosteroids in the treatment of community-acquired pneumonia in adults: a meta-analysis. PLoS One 2012; 7: e47926.

22 Tang BM, Craig JC, Eslick GD, et al. Use of corticosteroids in acute lung injury and acute respiratory distress syndrome: a systematic review and meta-analysis. Crit Care Med 2009; 37: 1594-1603.

23 Meduri GU, Marik PE, Chrousos GP, et al. Steroid treatment in ARDS: a critical appraisal of the ARDS network trial and the recent literature. Intensive Care Med 2008; 34: 61-69.

24 Ugajin M, Yamaki K, Hirasawa N, et al. Impact and indication of early systemic corticosteroids for very severe community-acquired pneumonia. Int J Gen Med 2013; 6: 693-701.

25 Salluh JI, Soares M, Coelho LM, et al. Impact of systemic corticosteroids on the clinical course and outcomes of patients with severe community-acquired pneumonia: a cohort study. J Crit Care 2011; 26: 193-200.

26 Chon GR, Lim CM, Koh Y, et al. Analysis of systemic corticosteroid usage and survival in patients requiring mechanical ventilation for severe community-acquired pneumonia. J Infect Chemother 2011; 17: 449-455.

27 Tagami T, Matsui H, Horiguchi $\mathrm{H}$, et al. Antithrombin and mortality in severe pneumonia patients with sepsis-associated disseminated intravascular coagulation: an observational nationwide study. J Thromb Haemost 2014; 12: 1470-1479.

28 American Thoracic Society, Infectious Diseases Society of America. Guidelines for the management of adults with hospital-acquired, ventilator-associated, and healthcare-associated pneumonia. Am J Respir Crit Care Med 2005; 171: 388-416.

29 Mandell LA, Wunderink RG, Anzueto A, et al. Infectious Diseases Society of America/American Thoracic Society consensus guidelines on the management of community-acquired pneumonia in adults. Clin Infect Dis 2007; 44 Suppl. 2, S27-S72.

30 Dellinger RP, Levy MM, Rhodes A, et al. Surviving sepsis campaign: international guidelines for management of severe sepsis and septic shock: 2012. Crit Care Med 2013; 41: 580-637.

31 Briel M, Bucher HC, Boscacci R, et al. Adjunctive corticosteroids for Pneumocystis jiroveci pneumonia in patients with HIV-infection. Cochrane Database Syst Rev 2006; 3: CD006150. 
Kim SH, Hong SB, Yun SC, et al. Corticosteroid treatment in critically ill patients with pandemic influenza A/ H1N1 2009 infection: analytic strategy using propensity scores. Am J Respir Crit Care Med 2011; 183: 1207-1214.

33 Rosenbaum PR, Rubin DB. Constructing a control-group using multivariate matched sampling methods that incorporate the propensity score. Am Stat 1985; 39: 33-38.

34 Stukel TA, Fisher ES, Wennberg DE, et al. Analysis of observational studies in the presence of treatment selection bias: effects of invasive cardiac management on AMI survival using propensity score and instrumental variable methods. JAMA 2007; 297: 278-285.

35 Adrie C, Schwebel C, Garrouste-Orgeas M, et al. Initial use of one or two antibiotics for critically ill patients with community-acquired pneumonia: impact on survival and bacterial resistance. Crit Care 2013; 17: R265.

36 Angus DC, van der Poll T. Severe sepsis and septic shock. N Engl J Med 2013; 369: 840-851.

37 Park DW, Chun BC, Kwon SS, et al. Red blood cell transfusions are associated with lower mortality in patients with severe sepsis and septic shock: a propensity-matched analysis. Crit Care Med 2012; 40: 3140-3145.

38 Wilson BZ, Anzueto A, Restrepo MI, et al. Comparison of two guideline-concordant antimicrobial combinations in elderly patients hospitalized with severe community-acquired pneumonia. Crit Care Med 2012; 40: 2310-2314.

39 Messika J, Sztrymf B, Bertrand F, et al. Risks of nonsteroidal antiinflammatory drugs in undiagnosed intensive care unit pneumococcal pneumonia: younger and more severely affected patients. J Crit Care 2014; 29: 733-778.

40 Staiger D, Stock JH. Instrumental variables regression with weak instruments. Econometrica 1994; 65: 557-586. 